Breast cancer is the most frequently diagnosed type of cancer in women worldwide. Both the development and progression of breast cancer are related to tumour evasion of the immune system through a process called cancer immune-editing, in which regulatory lymphocytes play an important role. The infiltration of Treg cells in patients with breast cancer has been proposed as an independent unfavourable prognostic factor. In the present study, we aimed to evaluate the percentages of the Treg cell populations in the peripheral blood of patients with breast cancer with respect to progesterone receptor expression. Peripheral blood samples were collected from 27 patients with breast cancer treated in the Clinical Department of Breast Cancer and Reconstructive Surgery of the Professor Franciszek Lukaszczyk Oncological Centre, Bydgoszcz. Flow cytometry was used to evaluate the percentage of CD25+l FOXP3+/CD127 (-/low) T cells within CD3+/CD4+ T cells.

The presence of CD25+/FOXP3+/ CD127 (-/low) T cells within CD3+/ $C D 4+T$ cells was identified in all the examined blood samples. A statistically significantly higher percentage of CD25+/FOXP3+/CD127 (-/low) T cells within $C D 3+/ C D 4+T$ cells was observed in progesterone receptor (PR)-negative breast cancer patients when compared to PR-positive breast cancer patients.

The observed high percentage of CD25+/FOXP3+/CD127 (-/low) T cells within $\mathrm{CD} 3+/ \mathrm{CD} 4+\mathrm{T}$ cells in PR $(-)$ breast cancer patients when compared to PR (+) breast cancer patients seems to confirm the unfavourable prognostic significance of these cells in breast cancer patients. This may indicate a rationale for combining standard oncological treatment in breast cancer patients with Treg-depleting therapy.

Key words: breast cancer, Treg lymphocytes, progesterone receptors.

Contemp Oncol (Pozn) 2018; 22 (4): 236-239 DOI: https://doi.org/10.5114/wo.2018.80038

\section{Analysis of Treg cell population in patients with breast cancer with respect to progesterone receptor status}

\author{
Konrad Dziobek ${ }^{1}$, Marta Biedka ${ }^{2,3}$, Tomasz Nowikiewicz ${ }^{4}$, \\ Maria Szymankiewicz ${ }^{5}$, Ewelina Łukaszewska ${ }^{6}$, Magdalena Dutsch-Wicherek ${ }^{5}$
}

${ }^{1}$ Department of Oncological Gynecology, Maria Sklodowska-Curie Memorial Cancer Centre and Institute of Oncology, Krakow Branch, Krakow

2Department of Radiotherapy, Professor Franciszek Lukaszczyk Oncology Center in Bydgoszcz, Poland

${ }^{3}$ Department of Oncology and Brachytherapy, Ludwik Rydygier Collegium Medicum in Bydgoszcz, Nicolaus Copernicus University in Torun, Bydgoszcz, Poland ${ }^{4}$ Clinical Department of Breast Cancer and Reconstructive Surgery, Professor Franciszek Lukaszczyk Oncology Center in Bydgoszcz, Poland

${ }^{5}$ Department of Oncology, Radiotherapy, and Gynecologic Oncology, Ludwik Rydygier Collegium Medicum in Bydgoszcz, Nicolaus Copernicus University in Torun, Bydgoszcz, Poland

${ }^{6}$ Department of Pathology, Professor Franciszek Lukaszczyk Oncology Center in Bydgoszcz, Poland

\section{Introduction}

Breast cancer is the most frequently diagnosed type of cancer in women and the second leading cause of death from cancer for women worldwide. The prognosis for breast cancer is determined by age, lymph node involvement, tumour grade, nuclear expression of estrogen receptor (ER), progesterone receptor (PR), and membrane expression of human epidermal growth factor receptor 2 (HER2) [1, 2]. The development and progression of the cancer is related to tumour evasion of the immune system through a process called cancer immune-editing consisting of three phases: elimination, equilibrium, and evasion. During the elimination phase, also called immunosurveillance, innate and adaptive immune responses cooperate to destroy the growing tumour cells before they become clinically apparent. In the equilibrium phase rare tumour cell variants that have escaped elimination outgrow. The cancer cells that have acquired resistance to the elimination enter the escape phase. During this phase cancer cells continue to grow and expand in an uncontrolled manner, enabled by several mechanisms including development of a suppressive cancer microenvironment. One if those mechanisms is recruitment of $T$ regulatory cells exerting a regulatory effect on the immune system. At this phase the tumour becomes clinically apparent. Among the tumour-infiltrating lymphocytes (TILS) in breast cancer patients are regulatory $T$ cells (Treg), which are identified by the nuclear factor forkhead box P3 (FOXP3) [3]. FOXP3+ TILs are correlated with a high risk of negative clinicopathological factors, such as ER negativity and high tumour grade $[4,5]$. Treg cell infiltration of breast cancer has been considered as an independent negative prognostic factor $[6,7]$. However, in a study conducted by West et al. with a cohort of 175 women with estrogen-receptor-negative breast cancers, FOXP3+ TILS were demonstrated as an independent positive prognostic factor in ER-negative breast cancer [8]. Tylor et al. demonstrated that the recruitment of Tregs to the cancer microenvironment inhibits an effective antitumour immune response, and in patients with claudin-low breast cancer, these tumours were found to be highly enriched with Tregs 
[9]. On the other hand, FOXP3+ TILs were also identified as an independent factor for improved survival and progression-free survival in triple-negative breast cancer [10].

In the present study, we aimed to evaluate the percentages of Treg cell populations in the peripheral blood of patients with breast cancer with respect to progesterone-receptor status.

\section{Material and methods}

The study included 27 patients who were treated surgically for breast cancer in 2017 in the Clinical Department of Breast Cancer and Reconstructive Surgery of the Lukaszczyk Oncological Centre, Bydgoszcz, Poland.

Patients were treated in line with the accepted management standard; in all cases, this involved combination treatment. All 27 patients underwent surgical treatment with radiotherapy of the breast. In every case, breast-conserving treatment $(\mathrm{BCT})$ with sentinel lymph node biopsy (SLNB) was applied [11, 12]. Each patient underwent radical surgery. According to current recommendations, this involved removal of the tumour within the limits of healthy tissues (no ink on the tumour) [11, 12], and this was confirmed by the histopathological examination of the state of surgical margins. The SLNB procedure identified the patients without the presence of metastatic lesions in the axillary (cNO group). The pre-operative assessment of the clinical condition required for this purpose included a physical examination of the patients supplemented by an ultrasound examination of the axilla. The isotope method was used to identify the sentinel lymph node. The surgical procedure was preceded by lymphoscintigraphy using $99 \mathrm{mTc}$ radionuclide with $75-100 \mathrm{MBq}$ activity on the albumin carrier (Nanocol). The isotopic marker was administered intradermally at the margin of the nipple envelope (in the breast quadrant where the primary change was located) approximately 2-3 hours before the surgery. For intraoperative identification of places of increased accumulation of radiotracers in the axillary cavity, and to measure the radiation value of the lymph nodes, a handheld gamma ray detector was used. The lymph node with the highest level of radiation was considered the sitter node sought during the surgical procedure. According to the " $10 \%$ rule" established by Martin et al. [13], lymph nodes displaying elevated radiotracer collection greater than $10 \%$ of the radiation value obtained for the sentinel node (nodes of the heart) were also removed.

The patient's consent was obtained in each case. Additionally, approval for the research program was granted by the Ethical Committee of the Nicolaus Copernicus University Ludwig Rydygier Collegium Medicum in Bydgoszcz (KBET/364/B/2015).

All the patients in our study had an invasive ductal breast cancer. From these patients two groups were selected: 19 patients with invasive breast cancer luminal type A: ER (+) PR (+) HER (-), Ki 67 until 15\% and eight patients with non-luminal (HER-positive) invasive breast cancer ER (-) PR (-) HER (+), Ki 67 in each case, according to Saint Gallen Consensus 2017. No statistically significant differences in tumour stage, lymph node status, tumour grade, and HER status were observed between the two groups of patients. The characteristics of the patient groups are presented in Table 1. From each of the patient peripheral blood samples were collected one day before the surgical procedure.

\section{Flow cytometry}

The samples for the cytometric evaluation of the Treg cell population in the whole blood of breast cancer patients (luminal A type) were prepared using Becton Dickinson reagents, according to the manufacturer's instructions. At first, the following antibodies for the detection of surface antigens were added to $100 \mu$ peripheral blood collected on EDTA: $5 \mu \mathrm{l}$ CD3 APC-Cy7, $20 \mu \mathrm{l}$ CD4 FITC, $20 \mu \mathrm{l}$ CD25

Table 1. Characteristics of the patient group

\begin{tabular}{|c|c|}
\hline Characteristic & Number of patients (\%) \\
\hline Age & 57 years (range: $31-64$ ) \\
\hline \multicolumn{2}{|l|}{ Tumour stage according to TNM 2010} \\
\hline $\mathrm{T} 1 \mathrm{~b}$ & $9(33)$ \\
\hline T1c & $17(62)$ \\
\hline $\mathrm{T} 2$ & $1(3)$ \\
\hline \multicolumn{2}{|l|}{ Lymph node status according to TNM 2010} \\
\hline Without lymph node involvement NO & $22(81)$ \\
\hline With lymph node involvement N1 & $5(19)$ \\
\hline \multicolumn{2}{|l|}{ Tumour differentiation grade } \\
\hline Grade 1 & $1(3)$ \\
\hline Grade 2 & $25(92)$ \\
\hline Grade 3 & $1(3)$ \\
\hline \multicolumn{2}{|l|}{ Histopatological assessment } \\
\hline Carcinoma ductale & $27(100)$ \\
\hline \multicolumn{2}{|l|}{ After operation margin } \\
\hline$>1 \mathrm{~cm}$ & $27(100)$ \\
\hline$<1 \mathrm{~cm}$ & $0(0)$ \\
\hline \multicolumn{2}{|l|}{ Hormonal status } \\
\hline ER positive & $19(70)$ \\
\hline ER negative & $8(30)$ \\
\hline PR positive & $19(70)$ \\
\hline PR negative & $8(30)$ \\
\hline \multicolumn{2}{|l|}{ HER status } \\
\hline HER positive & $8(30)$ \\
\hline HER negative & $19(70)$ \\
\hline \multicolumn{2}{|l|}{ Ki-67 expression } \\
\hline$<10 \%$ & $0(0)$ \\
\hline $10-15 \%$ & $19(70)$ \\
\hline$>20 \%$ & $8(30)$ \\
\hline \multicolumn{2}{|l|}{ Type of treatment } \\
\hline BCT operation & $27(100)$ \\
\hline Radiotherapy & $27(100)$ \\
\hline Brachytherapy & $26(96)$ \\
\hline Chemotherapy & $1(4)$ \\
\hline
\end{tabular}




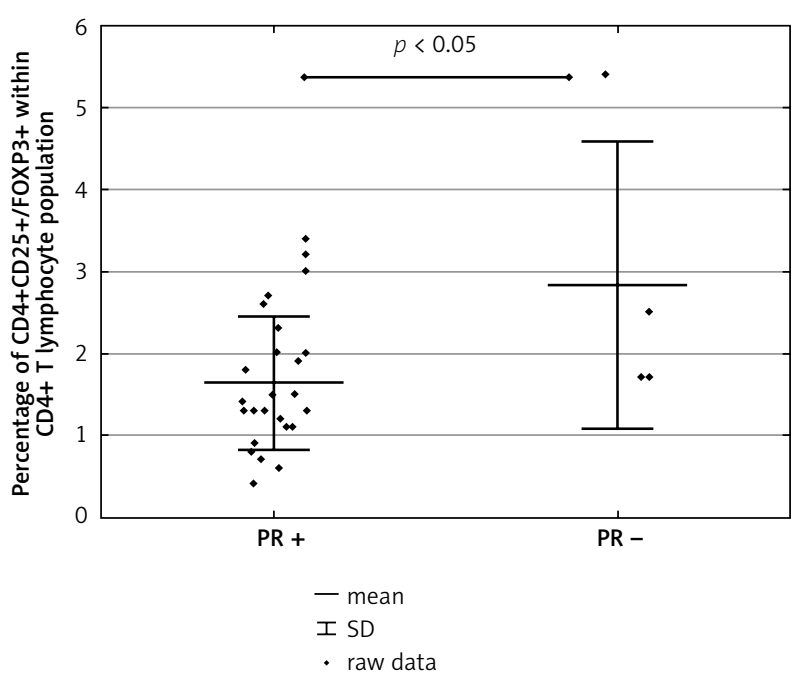

Fig. 1. The percentage of CD25+/FOXP3+/CD127 (-/low) T cells within CD3+/CD4+ $T$ cells in patients with progesterone receptor $(\mathrm{PR})(+)$ and PR (-) breast cancer tumours

APC, $5 \mu \mathrm{l} \mathrm{CD127} \mathrm{PerCP-Cy5.5,} \mathrm{and} 5 \mu \mathrm{l}$ CD194 PE-Cy7. After a 20-min incubation with mAbs (in the dark at room temperature - RT), the cells were washed with $2 \mathrm{ml}$ of fetal bovine serum (FBS) buffer and centrifuged for $10 \mathrm{~min}$ at $250 \times$ g. The obtained cell pellet was fixed with $2 \mathrm{ml}$ of freshly prepared $1 \times$ buffer A (for 10 min at RT) and centrifuged for $5 \mathrm{~min}$ at $500 \times \mathrm{g}$. The cells were then washed with $2 \mathrm{ml}$ of FBS buffer $(5 \mathrm{~min}, 500 \times \mathrm{g}$ ) and permeabilised with $0.5 \mathrm{ml}$ of freshly prepared buffer C (for 30 min in the dark at RT). After two further washing steps ( $2 \mathrm{ml} \mathrm{FBS;} 5 \mathrm{~min}$, $500 \times \mathrm{g}$ ), the cells were stained with $20 \mu \mathrm{l}$ of anti-human nuclear FOXP3 PE antibody for 30 min in the dark at RT. After two additional washing steps, the cells were suspended in $0.5 \mathrm{ml}$ of FBS buffer and analysed using BD FACS Canto II flow cytometer and BD FACS Diva Software (Becton Dickinson). In each sample, 15,000-25,000 CD3+/CD4+ cells were collected, among which the percentage of Treg lymphocytes was determined, according to the gating strategy described in the additional materials in the article by Santegoets et al. [14]. Treg cells were considered to be CD3+/CD4+/CD25+/ FOXP3+/CD127 (-/low).

\section{Statistical analysis}

The distribution of variables in the study groups of the women was checked with the use of the Shapiro-Wilk test. The statistical significance between the groups was determined by the Kruskal-Wallis test, one-way analysis of variance by ranks. The Mann-Whitney $U$ test was then used as applicable. All statistical analyses were carried out with Statistica 8.0 software (StatSoft Inc., Tulsa, OK, USA). A p-value $<0.05$ was considered indicative of statistical significance.

\section{Results}

The CD3+/CD4+/CD25+/FOXP3+/CD127 (-/low) T cells were identified in all the examined blood samples derived from the patients with breast cancer after the surgical pro- cedure. The statistically significantly higher percentage of CD25+/FOXP3+/CD127 (-/low) T cells within CD3+/CD4+ $T$ cells was observed in PR $(-)$ breast cancer patients following surgery when compared to PR (+) breast cancer patients following surgery (Fig. 1).

\section{Discussion}

The loss of PR may reflect a change to a more aggressive tumour behaviour [15]. PR is an ER-regulated gene that mediates the effects of progesterone on the development of mammary glands in physiology and is involved in the development of breast cancer. Increased breast cancer incidence is identified in patients after combined estrogen and progesterone hormone replacement therapy compared to estrogen therapy alone [16]. ER-positive/PR-negative metastatic tumours are observed to have a more aggressive course and are associated with a reduced overall survival compared to those preserving PR [17].

In the present study, statistically significantly higher CD25+/FOXP3+/CD127 (-/low) T cells within CD3+/CD4+ $T$ cells were identified in the patients with $\mathrm{PR}(-)$ breast cancers compared to those with $\mathrm{PR}(+)$ breast cancers. Moreover, in a meta-analysis, Wang et al. demonstrated that Treg cell infiltration was more prominent in breast cancer patients with ER (+) and PR (-) tumours. The loss of PR expression is correlated with more aggressive tumour behaviour and a reduced overall patient survival rate [18, 19]. In the presented study the number of the patients with PR-negative tumours is small, which might impair the statistics; however, the general trend in the presence of CD25+/FOXP3+/CD127 (-/low) T cells within the CD3+/ CD4+ $T$ cell population in PR (-) invasive breast cancer patients is documented.

Thus, it seems that patients with breast cancer might benefit from the elimination of Treg cells. Dendritic cells have been used in the development of antitumour vaccines because they trigger the antitumour immunity in cancer; however, this response was not sufficient to induce tumour regression. It has been proposed that this is due to the expansion of immunosuppressive cells such as Treg cells. In an experimental study by Ayala et al. [18], FOXP3 blockade improved the efficacy of dendritic cell vaccines by inhibiting Treg cells and the direct antitumour effect. It was thus proposed that FOXP3 blockade can neutralise the immunosuppressive tumour microenvironment and switch the antitumour immune response [18].

\section{Conclusions}

In conclusion, the subpopulation of regulatory $T$ cells in our study were related with the loss of PR expression, an unfavourable prognostic factor. Our study, together with other reports, therefore seems to confirm a rationale for combining the standard oncological treatment with Treg-depleting therapy.

The authors declare no conflict of interest. 


\section{References}

1. Nottegar A, Veronese N, Senthil M, et al. Extra-nodal extension of sentinel lymph node metastasis is a marker of poor prognosis in breast cancer patients: A systematic review and an exploratory meta-analysis. Eur J Surg Oncol 2016; 42: 919-925.

2. Bianchini G, Balko JM, Mayer IA, et al. Triple-negative breast cancer: challenges and opportunities of a heterogeneous disease. Nat Rev Clin Oncol 2016; 13: 674-690.

3. Burugu S, Asleh-Aburaya K, Nielsen TO. Immune infiltrates in the breast cancer microenvironment: detection, characterization and clinical implication. Breast Cancer 2017; 24: 3-15.

4. Mahmoud SM, Paish EC, Powe DG, et al. An evaluation of the clinical significance of FOXP3+ infiltrating cells in human breast cancer. Breast Cancer Res Treat 2011; 127: 99-108.

5. Droeser R, Zlobec I, Kilic E, et al. Differential pattern and prognostic significance of CD4+, FOXP3+ and IL-17+ tumor infiltrating lymphocytes in ductal and lobular breast cancers. BMC Cancer 2012; 12: 134.

6. Merlo A, Casalini P, Carcangiu ML, et al. FOXP3 expression and overall survival in breast cancer patients. J Clin Oncol 2009; 27: 1746-1752.

7. Bates GJ, Fox SB, Han C, et al. Quantification of regulatory T cells enables the identification of high-risk breast cancer patients and those at risk of late relapse. J Clin Oncol 2006; 24: 5373-5380.

8. West NR, Kost SE, Martin SD, et al. Tumor-infiltrating FOXP3+ lymphocytes are associated with cytotoxic immune responses and good clinical outcome in oestrogen receptor-negative breast cancer. Br J Cancer 2013; 108: 155-162.

9. Lee S, Cho EY, Park YH, et al. Prognostic impact of FOXP3 expression in triple-negative breast cancer. Acta Oncol 2013; 52: 73-81.

10. Yeong J, Thike AA, Lim JC, et al. Higher densities of Foxp3+ regulatory $T$ cells are associated with better prognosis in triple-negative breast cancer. Breast Cancer Res Treat 2017; 163: 21-35.

11. Taylor NA, Vick SC, Iglesia MD, et al. Treg depletion potentiates checkpoint inhibition in claudin-low breast cancer. J Clin Invest 2017; 127: 3472-3483

12. Coates AS, Winer EP, Goldhirsch A, et al. Tailoring therapies-improving the management of early breast cancer: St Gallen International Expert Consensus on the Primary Therapy of Early Breast Cancer 2015. Ann Oncol 2015; 26: 1533-1546.

13. National Comprehensive Cancer Network (2016). National Comprehensive Cancer Network (NCCN) Clinical Practice Guidelines in Oncology: Breast Cancer Version 1.2016. Retrieved 28 March 2016 at www.ncen.org.

14. Martin RC, Edwards MJ, Wong SL, et al. Practical guidelines for optimal gamma probe detection of sentinel lymph nodes in breast cancer: results of a multi-institutional study. For the University of Louisville Breast Cancer Study Group. Surgery 2000; 128: 139-144.

15. Santegoets SJ, Dijkgraaf EM, Battaglia A, et al. Monitoring regulatory $T$ cells in clinical samples: consensus on an essential marker set and gating strategy for regulatory $T$ cell analysis by flow cytometry. Cancer Immunol Immunother 2015; 64: 1271-1286.

16. Broom RJ, Tang PA, Simmons C, et al. Changes in estrogen receptor, progesterone receptor and Her-2/neu status with time: dis cordance rates between primary and metastatic breast cancer. Anticancer Res 2009; 29: 1557-1562.

17. Rossouw JE, Anderson GL, Prentice RL, et al. Risks and benefits of estrogen plus progestin in healthy postmenopausal women principal results from the women's health initiative randomized controlled trial. JAMA 2002; 288: 321-333.

18. Franco A, Col N, Chlebowski RT. Discordance in estrogen (ER) and progestin receptor (PR) status between primary metastatic breast cancer: A meta-analysis. J Clin Oncol 2004; 22 (Suppl): 539-539.

19. Wang Y, Sun J, Zheng R, et al. Regulatory T cells are an important prognostic factor in breast cancer: a systematic review and meta-analysis. Neoplasma 2016; 63: 789-798.

20. Ayala MA, Gottardo MF, Imsen M, et al. Therapeutic blockade of Foxp3 in experimental breast cancer models. Breast Cancer Res Treat 2017; 166: 393-405.

\section{Address for correspondence}

\section{Magdalena Dutsch-Wicherek}

Department of Oncology, Radiotherapy, and Gynecologic Oncology Ludwik Rydygier Collegium Medicum in Bydgoszcz

Nicolaus Copernicus University in Torun

2 Romanowskiej St.

85-796, Bydgoszcz, Poland

e-mail: mowicher@gmail.com

Submitted: 14.09 .2018

Accepted: 24.10.2018 